\title{
THE CHARACTERISTICS OF WATER DROPLET EVAPORATION IN THE FLAMES OF VARIOUS FLAMMABLE LIQUIDS
}

\author{
Margarita A. Dmitrienko, Alena O. Zhdanova ${ }^{a}$, Galina S. Nyashina \\ National Research Tomsk Polytechnic University, 634050 Tomsk, Russia
}

\begin{abstract}
This paper investigates the integral characteristics of the evaporation of water droplets (the initial diameter 4-6 mm) during their motion in flames obtained through the combustion of typical flammable liquids for various purposes, such as kerosene and denatured alcohol. We performed the experiments using high-speed video cameras and panoramic cross-correlation techniques PTV and SP. The difference between evaporation rates under appropriate conditions has been defined. The influence of a droplet initial size on evaporation rate has been demonstrated.
\end{abstract}

\section{Introduction}

To date, various combustible liquids, gas, solid and liquid fuels are used to generate high-temperature gases in modern thermal water treatment systems. In practice, liquid fuels and various specialized flammable liquid compounds are used most commonly. The modern systems of thermal water treatment have a rather low efficiency. Recent reports (for example, [1]) concluded that this is due to the fact that the laws of the evaporation of water droplets, emulsions and suspensions in hightemperature gases have not been fully investigated. This is due to the high complexity of considered processes and the need to apply the most advanced experimental techniques for their investigation. In particular, papers [2,3] present the study of water droplet evaporation in kerosene combustion products. The investigations [2, 3] were performed using panoramic optical flow visualization techniques (PIV, IPI, PTV) [4, 5]. However, it has not yet been analyzed, whether the established integral characteristics of water evaporation when burning kerosene correspond to the processes when burning other flammable liquids commonly used in thermal water treatment. Therefore, it is advisable to analyze the processes of water evaporation when burning the most widely used fuels, such as kerosene and denatured alcohol.

The aim of this paper is to compare the integral characteristics of water droplet evaporation in the combustion products of various flammable liquids on the example of kerosene and denatured alcohol.

\section{Experimental Setup and Procedure}

Fig. 1 shows a scheme of our experimental setup. The setup provided a video recording of single water droplets passing the distance of $1 \mathrm{~m}$ from a dosing devise 12 to a catcher 14 .

a Corresponding author: zhdanovaao@tpu.ru 


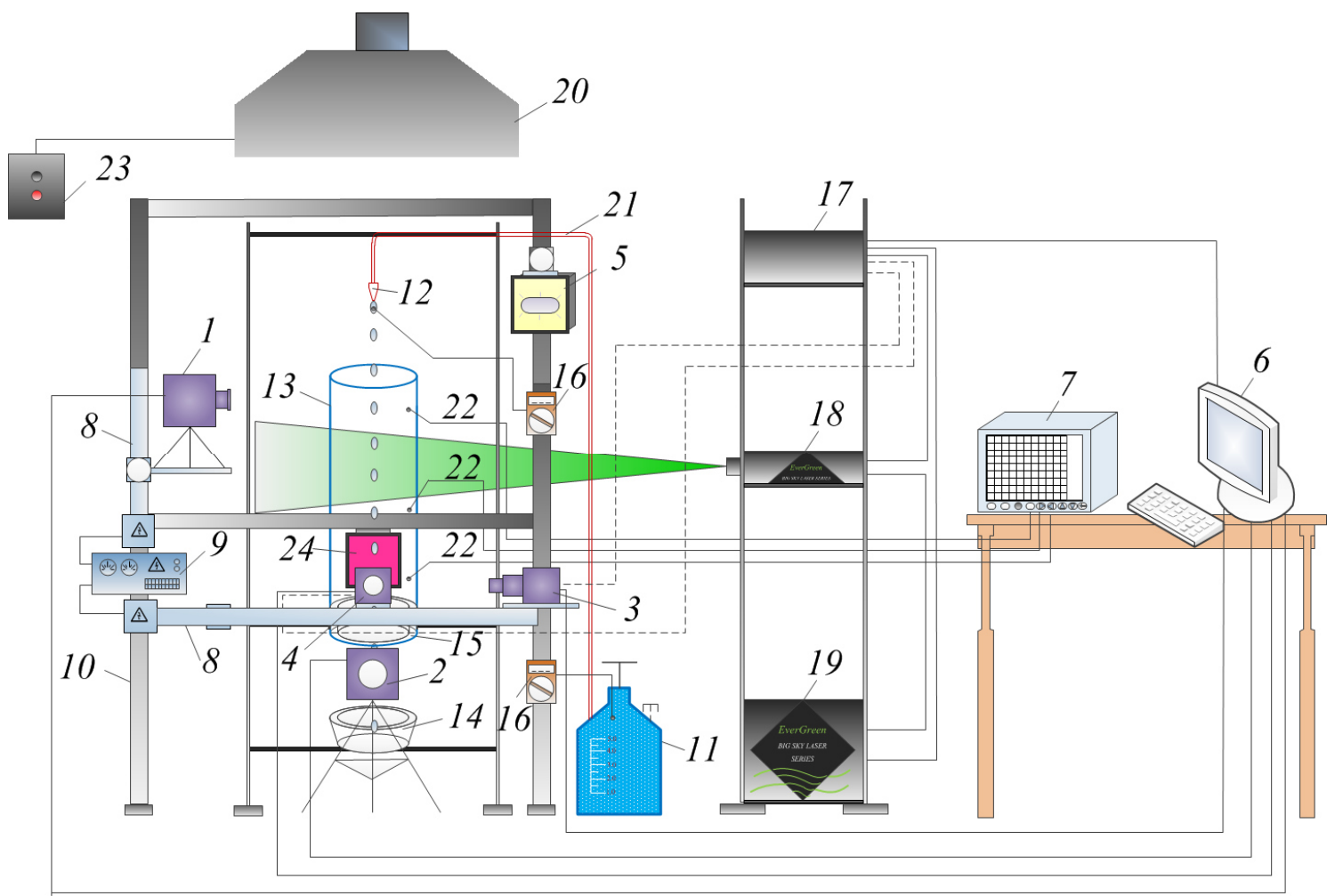

Figure 1. Scheme of an experimental setup: 1,2 - high-speed video cameras; 3,4 - cross-correlation video cameras; 5 - spotlight; 6 - personal computer; 7 - multichannel technological registrar; 8 - motorized pointing device; 9 - power supply for multichannel technological registrar; 10 - aluminum rack; 11 - water tank; 12 dosing devise; 13 - quartz tube; 14 - catcher; 15 - hollow tube with flammable liquid; 16 - digital multimeter; 17 - synchronizer of computer, cross-correlation cameras and laser; 18 - double-pulse laser; 19 - laser line generator; 20 - exhaust hood, 21 - water supply tubing; 22 - thermocouples; 23 - remote on/off switch; 24 diffusion screen with a light guide.

The initial size of water droplets $\left(d_{0}\right)$ varied from 4 to $6 \mathrm{~mm}$, their velocity $\left(u_{0}\right)$ varied from 2 to 4 $\mathrm{m} / \mathrm{s}$. The dosing devise 12 released droplets with the initial velocity $u_{0}$; the velocity of water droplets increased up to $u=5 \mathrm{~m} / \mathrm{s}$ after passing $1 \mathrm{~m}$.

Our experimental setup (Fig. 1) included two high-speed video cameras 1, 2 (frame frequency is up to $6 \cdot 10^{5} \mathrm{fps}$ ), a registration complex based on cross-correlation video cameras 3 and 4 , a doublepulse laser 18 , a signal synchronizer 17 , a laser line generator 19 and a specialized workstation 6 (for data processing). We determined the sizes of droplets during their movement at speeds in excess of 2 $\mathrm{m} / \mathrm{s}[6,7]$ with the help of software "Tema Automotive". Software "Tema Automotive" allowed us to monitor continuously the shape of a droplet.

The internal cavity of a cylindrical channel 13 was filled with flammable liquid (kerosene or denatured alcohol), which was then ignited. As a result, the entire inner cavity of a cylindrical channel 13 was filled with high-temperature gas. After about 200 seconds (time delay was necessary to achieve the desired gas temperature in the cylindrical channel 13), water was fed from the tank 11 to the input of the dosing device 12 via tubing 21. Droplets were realeased from the dosing devise 12 to the cylindrical channel 13 (droplets moved along the symmetry axis of the cylindrical channel from the top down). Video cameras 1 and 2, as well as cross-correlation video cameras 3 and 4 recorded droplets in free fall in high-temperature gas. Video recordings were transferred to the workstation 6 , where we performed their processing by means of software "Tema Automotive". 
We carried out at least 10 series of experiments under identical initial conditions (the initial diameter of droplets $d_{0}$, the initial temperature of droplets $t_{0}$, the initial velocity of droplets $u_{0}$, gas (combustion products) temperature $t_{\mathrm{g}}$, gas velocity $u_{\mathrm{g}}$ ).

Gas temperature $T_{\mathrm{f}}$ (of combustion products) ranged from $305 \mathrm{~K}$ to $1652 \mathrm{~K}$ in the experiments. Measurements were performed using three tungsten-rhenium thermocouples 22 (the range of temperature measurement $0 \div 2473 \mathrm{~K}$, permissible variation $\pm 0,005 T_{\mathrm{f}}$ ). The initial temperature of water droplets released into gas was maintained around $300 \mathrm{~K}$ by heating chambers. This temperature was recorded by chromel-copel thermocouple (the range of measured temperatures 233-573 K, permissible variation $\Delta=2.5 \mathrm{~K}$ ) with a multimeter 16 in the complete set. Gas velocity $U_{\mathrm{g}}$ inside the cylindrical channel was about $1.5 \mathrm{~m} / \mathrm{s}$. We conducted the measurements of $U_{\mathrm{g}}$ by the panoramic optical method PIV. Before carrying out experiments with water droplets, we sprinkled "tracers" - the particles of titanium dioxide, into gas environment. The speed of "tracers" and gas was maintained at about $1.5 \mathrm{~m} / \mathrm{s}$. The dosing rate of water droplets was maintained at a value 1 drop per second.

We determined four maximum diameters (in pixels) of a water droplet before and after the flame zone. Droplet sizes were determined from a video, which was a consistent set of video frames at a fixed time interval. Then, we calculated the average diameter: $d_{1}=\left(d_{01}+d_{02}+d_{03}+d_{04}\right) / 4$. The same procedure was performed for subsequent droplets: $d_{2}=\left(d_{01}+d_{02}+d_{03}+d_{04}\right) / 4$. After that, we found the average diameter of all droplets $d_{\text {drop(pix) }}=\left(d_{1}+d_{2}+. .+d_{\mathrm{n}}\right) / \mathrm{n}$. We converted pixels into millimeters at a certain (predetermined) scale factor $\mathrm{S}(\mathrm{mm} / \mathrm{pixel}): d_{\mathrm{drop}(\mathrm{mm})}=d_{\mathrm{drop}(\mathrm{pix})} \times S$, and then calculated the corresponding values of the average droplet radius. Thereafter, we calculated a parameter, which characterizes the size reduction of droplets during their movement through high-temperature gas environment: $\Delta R=\left(R_{\mathrm{d}}-R_{\mathrm{d}}{ }^{*}\right) / R_{\mathrm{d}}$, where $R_{\mathrm{d}}, R_{\mathrm{d}}{ }^{*}$ - the conditional average radius of a droplet at the input and output zones of high-temperature gases, $\mathrm{mm}$. The systematic measurement errors of high-speed video cameras 1 and 2 did not exceed $0.05 \div 0.08 \mathrm{~mm}$ at $S=0.041 \div 0.055$.

The processing of video frames by means of software "Phantom Camera Control" involved the measurement of the size and speed of moving droplets. To evaluate them, we observed the position of a droplet at the initial and final time. Then, we calculated droplet velocity, knowing a pre-measured scale factor and a certain time interval between the positions of the droplet in the video.

\section{Results and discussion}

Fig. 2 shows several typical images of droplets when $d_{0}=5.6 \mathrm{~mm}$ and $u_{0}=2.1 \mathrm{~m} / \mathrm{s}$ at the input to the cylindrical channel $13(a-c)$ and at the output after passing the channel with denatured alcohol combustion products $(d-f)$.

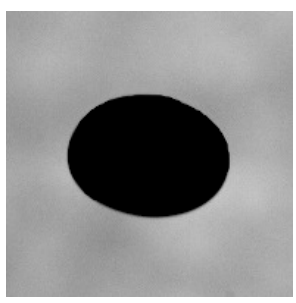

$a$

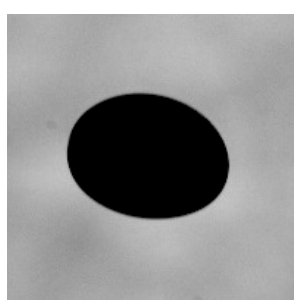

b

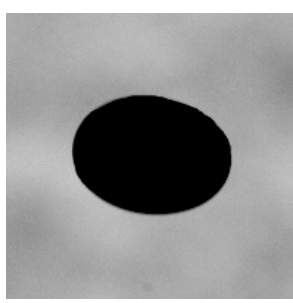

c 


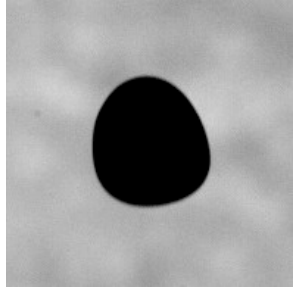

$d$

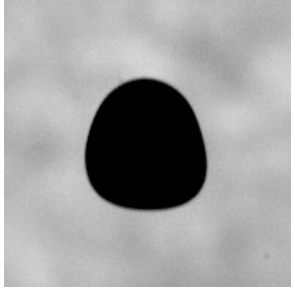

$e$

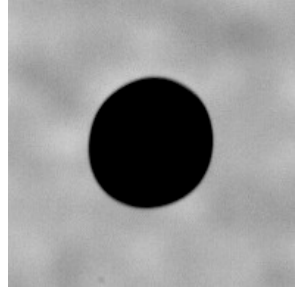

$f$

Figure 2. Typical images of water droplets at the input $(a-c)$ and output $(d-f)$ of denatured alcohol combustion products at $d_{0}=5.6 \mathrm{~mm}$ and $u_{0}=2.1 \mathrm{~m} / \mathrm{s}$.

Fig. 3 shows the shapes of droplets during their movement in the flame obtained through the combustion of denatured alcohol.

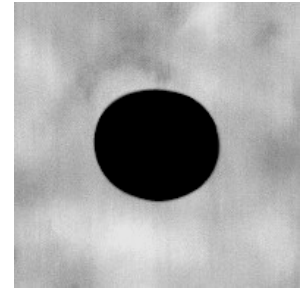

$a$

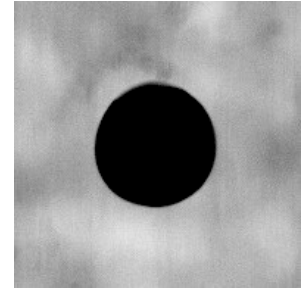

$b$

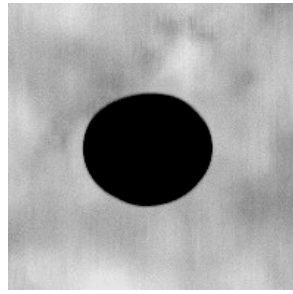

$c$

Figure 3. Typical images of water droplets in the flame obtained through the combustion of denatured alcohol; $d_{0}=5.6 \mathrm{~mm}$ and $u_{0}=2.1 \mathrm{~m} / \mathrm{s}$.

Table 1 shows the temperature distribution of combustion products over the entire height of the cylindrical channel 13. Gas temperature $T_{\mathrm{f}}$ (of combustion products) ranged from $305 \mathrm{~K}$ to $1652 \mathrm{~K}$ for denatured alcohol and from $397 \mathrm{~K}$ to $1611 \mathrm{~K}$ for kerosene. Measurements were carried out at the height from $0.1 \mathrm{~m}$ to $1 \mathrm{~m}$ relative to the base of the cylindrical channel 13 . The flame temperature of denatured alcohol exceeded the flame temperature of kerosene only at the height of $0.1 \mathrm{~m}$; at a higher height the flame temperature of denatured alcohol was considerably lower.

Table 1 presents data on the different heating degrees of droplets during their movement in hightemperatures obtained through the combustion of typical flammable liquids.

Table 1. Temperatures of denatured alcohol and kerosene combustion products in a cylindrical channel

\begin{tabular}{|l|l|l|l|l|l|}
\hline The position of a thermocouple relative to the base of a channel & $0.1 \mathrm{~m}$ & $0.3 \mathrm{~m}$ & $0.5 \mathrm{~m}$ & $0.7 \mathrm{~m}$ & $1 \mathrm{~m}$ \\
\hline Denatured alcohol & 1652 & 796 & 530 & 405 & 305 \\
\hline Kerosene & 1611 & 879 & 627 & 502 & 397 \\
\hline
\end{tabular}

The results of experimental studies established a decisive influence of a droplet size on the intensity of droplet heating and evaporation in a stream of high-temperature combustion products. Table 2 shows the values of parameters $\Delta R$ and $R_{\mathrm{d}}$ for different initial velocities of droplets. It is assumed that droplets move in the stream of high-temperature combustion products of typical flammable liquids for various purposes - kerosene and denatured alcohol.

Table 2. Values of $\Delta \mathrm{R}(\%)$ for kerosene and denatured alcohol depending on the initial size and initial velocity of a droplet

\begin{tabular}{|l|c|c|c|c|c|c|c|c|c|}
\hline Initial velocity $u_{0}, \mathrm{~m} / \mathrm{s}$ & \multicolumn{3}{|c|}{2} & \multicolumn{3}{|c|}{3} & \multicolumn{3}{|c|}{4} \\
\hline Initial droplet size $R_{\mathrm{d}}, \mathrm{mm}$ & 2 & 2.5 & 3 & 2 & 2.5 & 3 & 2 & 2.5 & 3 \\
\hline Denatured alcohol & 5.6 & 4.2 & 3.1 & 5.5 & 3.9 & 2.7 & 5.3 & 3.6 & 2.4 \\
\hline
\end{tabular}




\begin{tabular}{|l|l|l|l|l|l|l|l|l|l|}
\hline Kerosene & 7.5 & 5.6 & 4.1 & 7.3 & 5 & 3.5 & 6.9 & 4.4 & 2.8 \\
\hline
\end{tabular}

Results demonstrated that the droplet size $2<R_{\mathrm{d}}<3 \mathrm{~mm}$ decreased, on average, by $5.6 \%$, when droplet passed the distance of $1 \mathrm{~m}$ in a high-temperature gas stream of denatured alcohol combustion products. A similar parameter was $7.5 \%$ for droplets passed the same distance in the flame of kerosene. Thus, the relationship between the proportion of evaporated water $\Delta R$ and droplet size is significantly non-linear at the constant initial sizes and velocities of droplets.

Such results can be explained by the fact that an increase in a water droplet initial size causes an increase in time required for heating a droplet subsurface layer to the temperature of intensive evaporation. Therefore, the evaporation of droplets is considerably less intensive than that of droplets with the initial radius of $2 \mathrm{~mm}$. The increase of the initial velocity $\left(u_{0}\right)$ of droplets affects time for their passing the distance of $1 \mathrm{~m}$ in the high-temperature stream of flammable liquids combustion products. This explains a moderate reduction of $\Delta R$ at the initial velocity $4 \mathrm{~m} / \mathrm{s}$, compared to that for $2 \mathrm{~m} / \mathrm{s}$.

Experiments proved that the variation of the initial size and velocity of water droplets considerably affects the characteristics of their heating and evaporation in high-temperature gas stream obtained through the combustion of common flammable liquids used in thermal wastewater treatment. Also, the temperature of flammable liquids combustion has a determining influence on evaporation rate. When droplets pass the distance of $1 \mathrm{~m}$ in kerosene combustion products, the value of $\Delta R$ increases significantly compared to that in denatured alcohol combustion products. This corresponds well to data presented in Table 1.

It should be noted that the characteristic values of the integral parameter $\Delta R$ correlate well with the results of numerical simulation [8]. In particular, author [8] concluded that the evaporation rate of water droplets is nonlinearly dependent on gas temperature. According to the results of numerical simulation [8], the maximum evaporation rate is $0.241 \mathrm{~kg} /\left(\mathrm{m}^{2} \cdot \mathrm{s}\right)$. If we consider the results of the experiments conducted at gas temperature 1000-1200 K (according to [8]), then, with the help of video recording tools, it is possible to find the values of $\Delta R$ and the characteristic times of droplets passing through high-temperature environment. By the method described in study [9], we found that evaporation rates ranged from $0.05 \mathrm{~kg} /\left(\mathrm{m}^{2} \cdot \mathrm{s}\right)$ to $0.27 \mathrm{~kg} /\left(\mathrm{m}^{2} \cdot \mathrm{s}\right)$ in our experiments. This means that the results of our experiments correspond to the results obtained in numerical simulation. Therefore, models, suggested in study [8], can be used as prognostic models for determining the dynamic of the change in the size of droplets during their evaporation in high-temperature gases. In practice, this is especially important for gas vapor-droplet technologies in security sector, power and chemical industry.

\section{Conclusions}

Experiments showed that the integral characteristics of water evaporation may vary significantly, when water droplets pass through combustion products of common flammable liquids, which are used in wastewater treatment systems. This is mainly due to the temperature of gases, and consequently, the temperature of droplets. Under high-temperatures, the change of even a few percent of heat flow to a droplet may lead to a significant increase or decrease of the integral characteristics of evaporation. This is due to the nonlinear dependence of evaporation rate from temperature.

Highlighted regularities allow us to conclude that the efficiency of modern water treatment systems can be significantly increased only by the individual approach to the choice of parameters for the equipment with different high-temperature combustion products. In particular, prognostic models [8] may be used. This allows obtaining results, which correlate well with data of experiments.

The study was financially supported by the grant of Russian Foundation for Basis Research (project 15-38-20006). 


\section{References}

1. A.Yu. Varaksin, High Temp. 51, 377 (2013).

2. R.S. Volkov, O.V. Vysokomornaya, G.V. Kuznetsov, P.A. Strizhak, J. Eng. Phys. Thermophys. (Other) 86, 1413 (2013).

3. R.S. Volkov, G.V. Kuznetsov, P.A. Strizhak, J. Eng. Phys. Thermophys. (Other) 87, 450 (2014).

4. J.V. Simo Tala, S. Russeil, D. Bougeard, J.-L. Harion, Exp. Therm. and Fluid Sci. 50, 45 (2013).

5. D. Damiani, E. Meillot, D. Tarlet, J. Therm. Spray Technol. 23, 340 (2014).

6. J. Janiszewski, Metrol. and Measur. Sys. 19, 797 (2012).

7. J. Janiszewski, Int. J. Solids and Struct. 49, 1001 (2012).

8. P.A. Strizhak, J. Eng. Phys. Thermophys. (Other) 86, 895 (2013).

9. G.V. Kuznetsov, P.A. Kuibin, P.A. Strizhak, High Temp. 53, 254 (2015). 